\title{
FPGA Implementation of Reversible Medical Image Watermarking
}

\author{
Dr. Ahlam Fadhil Mahmood \\ Computer Engineering Department \\ University of Mosul \\ ahlam.mahmood@gmail.com
}

\begin{abstract}
Medical image protection and authentication are becoming increasingly important in an e-Health environment where images are readily distributed over electronic networks.

This paper presents an FPGA implementation of reversible watermarking encoder and decoder system. The system is based on the Discrete Cosine Transform (DCT/IDCT) to embed and extract the copyright protection mark and least significant bit (LSB) technique to hide the patients' information and then extract back the information by the owner using.

The proposed structure employs a single multiplierless 1D-DCT/IDCT block, instead of three in many existing DCT watermarking systems, which is reduce the hardware DCT part to $16.6 \%$ compared with previous proposals. The parallel hardware implementation of DCT and LSB is done in Xilinx XSC3S500 FPGA. The proposed scheme allows multi-insertions by many doctors in order to give an exact diagnosis to the patient.
\end{abstract}

portable system of the watermarking with small size and low power dissipation Keywords: Information hiding; watermarking ; FPGA; DCT/IDCT; Least Significant Bit method.

$$
\begin{aligned}
& \text { رقاقة البوابات القابلة للبرمجة حقليا لتتفيذ العلامة المائية وأسترجاعها للصور الطبية } \\
& \text { د.أحلام فاضل محمود } \\
& \text { قسم هندسة الحاسوب } \\
& \text { ahlam.mahmood@gmail.com }
\end{aligned}
$$

أن حماية وتحقيق ملكية الصور الطبية أُصبحت مهمة جداً في بيئةِ الصحةِ إلاكترِنيةِ حيث تنقل الصورِ بسهولة

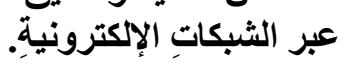

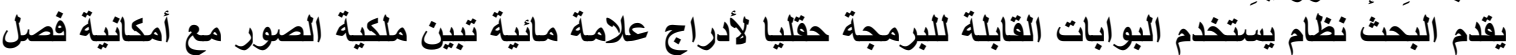

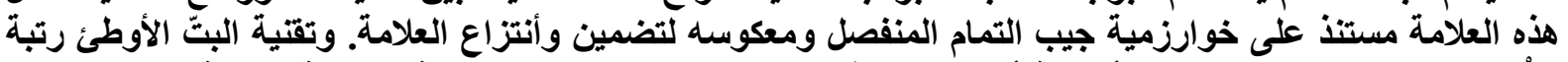

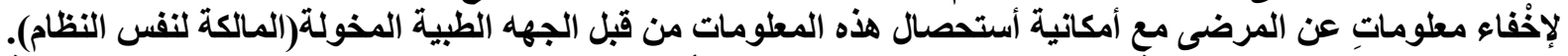

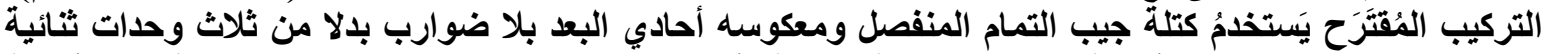

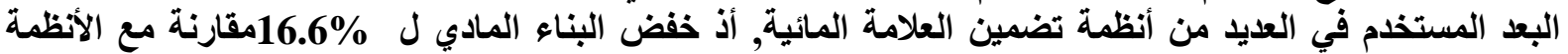

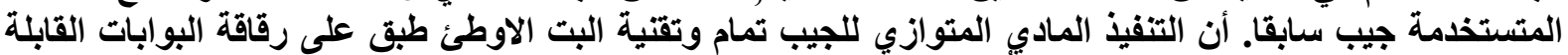

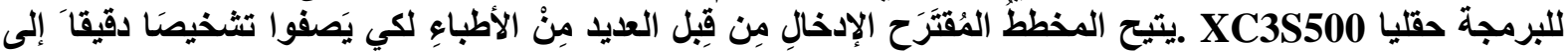




\section{Introduction}

Watermarking is a process by which a discrete data stream called a watermark is hidden within a host signal by imposing imperceptible changes on the signal[1]. Major applications of watermarking include copyright protection and Hiding information[2].

Among different kinds of digital watermarking schemes, reversible watermarking has become a research hotspot recently[3][4]. Compared with traditional watermarking, it can restore the original cover media through the watermark extracting process; thus, reversible watermarking is very useful, especially in applications dictating high fidelity of multimedia content, such as military aerial intelligence gathering, medical records, and management of multimedia information. Hiding patient data in the medical image is one of the applications of digital image watermarking. The patient data in the electronic format is called Electronic patient record (EPR)[5].

Most of the existing watermarking algorithms are designed for software implementations in general-purpose computers [6]. However, some applications, such as HDV broadcast monitoring and on-air live broadcasting, may require a portable system of the watermarking with small size and low power dissipation, which creates the need for hardware implementation of the watermarking. Some work about circuit implementation has been reported in [7]. These architectures are implemented by mapping a DCT watermarking algorithm in a two 2-D DCT structure that are performed in parallel. Due to the large amount of the computation of the watermarking process, this kind of architectures usually requires a large number of basic blocks [8].

Another medical image watermarking system embeds is the patient information such as patient details, history and measured physiological signals in the Least Significant Bit (LSB) plane [9].

In this paper, a reversible watermarking system is proposed; the proposed scheme is mainly composed of two parts for the embedding of the watermark. First: the copyright image is based on new computation of the Discrete Cosine Transform in frequency domain. Second: the patient information embedded/extractor is based on LSB information hiding algorithm.

The rest of the paper is organized as follows. The copyright protection frequency domain issues of the proposed watermarking scheme are described in Section 2.1, in Section 2.2 the LSB replacement are present . Experimental results are given in section 2.3. Then the details of the FPGA proposed algorithm including the embedding and extracting processes are elaborated in Section 3. The hardware implementation results are given and analyzed in Section 4. Finally, conclusions are drawn in Section 5.

\section{The Proposed Watermarking}

In this paper, The propose scheme is composed of two parts. The first one is to embed and extract the patient's data (name, job; age, gender; mobile, hospital registrations; and diagnosis) in the medical image and the second is about how to embed logo watermarking schemes which are needed for copyright protection.

An important factor to be considered while watermarking medical images is that medical images contain Region of Interest (ROI) and Regions of Non-Interest (RONI). In medical images, ROI is an area that contains diagnostically important information and must be processed without any distortion. The watermark is usually being embedded in the RONI (Region Of Non Interest) as this region does not contribute in the process of diagnosis[11]. Thresholding, Otsu's method are used, which chooses the threshold to minimize the interclass variance of the black and white pixels, behind segmentations, the following points record the operations of the proposed algorithm: 
1. Read X-ray and logo image.

2. At First, a threshold value is defined to distinguish between the region of interest (ROI) and the background area of the image (RONI). All pixel values greater than the threshold value, are considered as belongs to the region of interest or else belongs to the background.

3. Search in RONI region to find first same logo size window.

4. Use frequency domain Discrete Cosine Transformation to embed logo image. The DCT of both the images are taken and the proportion in which the DCT is added can vary the intensity of the image that will appear as the watermark.

5. Initialize the RONI area to embed patient information at the end of logo insertion region, divide the remainder RONI area into five field, first field to insert patient Name and job; Second field content gender, mobile and age ; , Third field to embed the hospital record information; fourth field hide the first doctor diagnosis;. The last field allows multi-insertions by many doctors in order to give an exact diagnosis to the patient. Each field are ended using ";" mark, the last field space is all the reminder RONI LSB bits.

6. Save the obtained image and stored as report in Electronic patient record (EPR) and it can be transmit to any other hospital to embed new diagnosis.

7. The receiver hospital whose have the decoder system can extract the logo image after read key from received image by applying Inverse transform.

8. Extract the first four field by inverse LSB method with convert the binary bits to ASCII code and save it as text file.

9. The new doctors in received hospital can embed new case diagnosis as text and enable hardware system to hide it in last field. The embedded and extract schemes are verified in the following flow charts.

\subsection{The First Proposed Watermarking Scheme Based on DCT/IDCT:}

One of the most commonly used watermarking procedures involving the 2-D DCT [7][8][10]. The basic process that is taking place is the addition of DCTs followed by the IDCT of the result which gives the watermarked image [7].

$V_{i}^{s}=V_{i} *\left(1+\alpha *\left(X_{i}+\beta * W_{i}\right)\right.$

$V_{i}^{\prime}$ is the result of the added 2D-DCT of the two images. $X_{i}$ is the 2D-DCT value of the image on which the watermarking is done and $W_{i}$ is the 2D-DCT of the logo which is watermarked on the image. The constants $\alpha$ and $\beta$, decides the visibility of the watermark. If the value of $\beta$ is very small, we obtain an invisible watermark and as its value increases the visibility simultaneously increases.

For the extraction of a watermark, the value of $\alpha$ and $\beta$ has to be known, hence, the extraction of the watermarking cannot be done by anyone who does not know those values.

As shown in Figure 2.a, there are two 2-D DCT computations which can be performed in parallel [7][10]. One is for the original image $x$ and the other for the watermark $w$. Most literature architectures are implemented by mapping a DCT watermarking algorithm in a structure in which the 2-D DCT of the signal and that of the watermark are performed in parallel. 


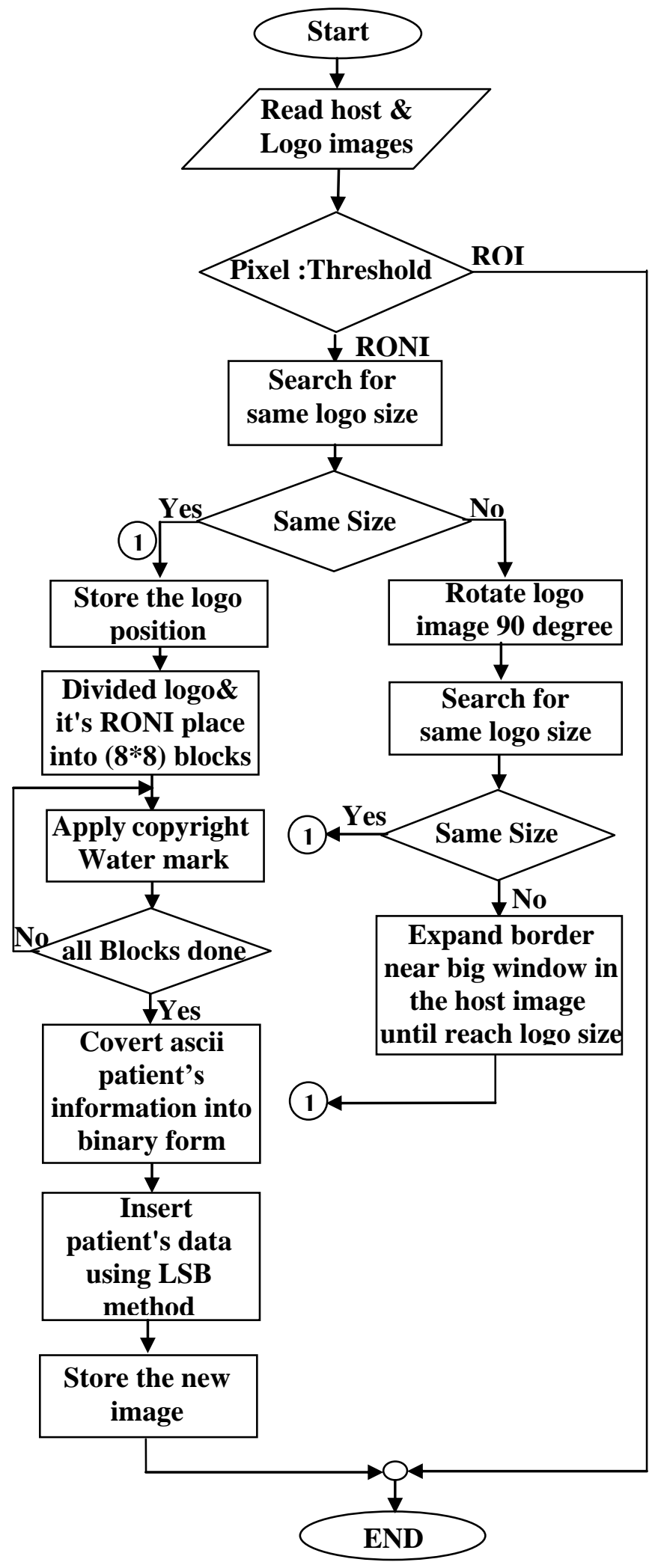

(a) Embedder

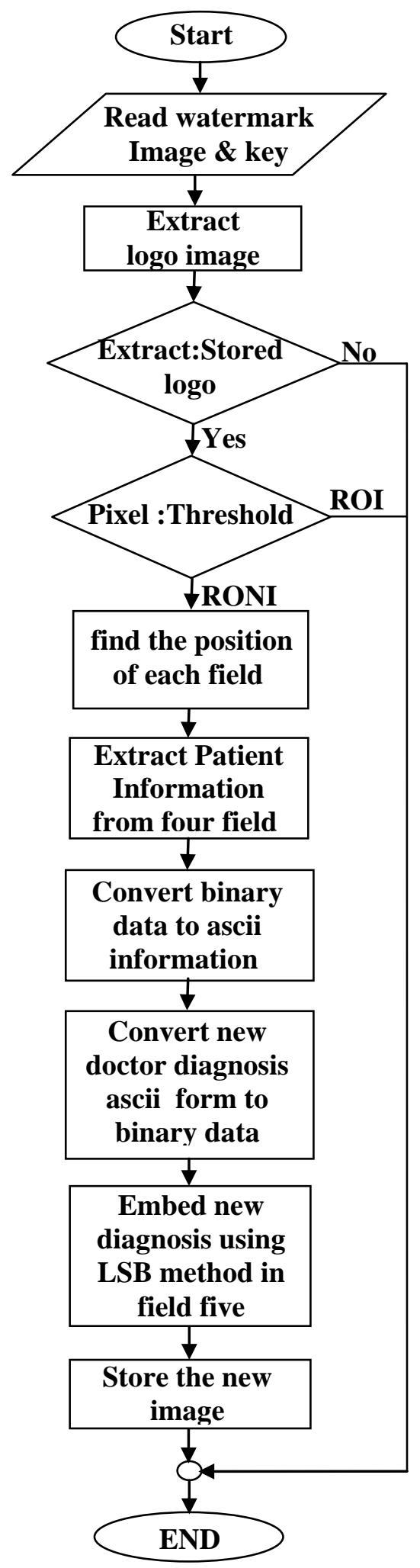

(b) Extractor

Figure 1: Flow Chart of the Proposed System 


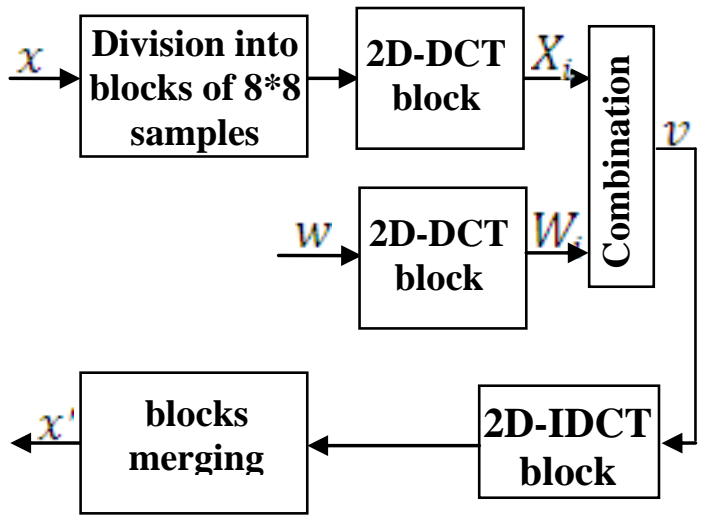

(a) General scheme of the Watermarking using the 2D- DCT

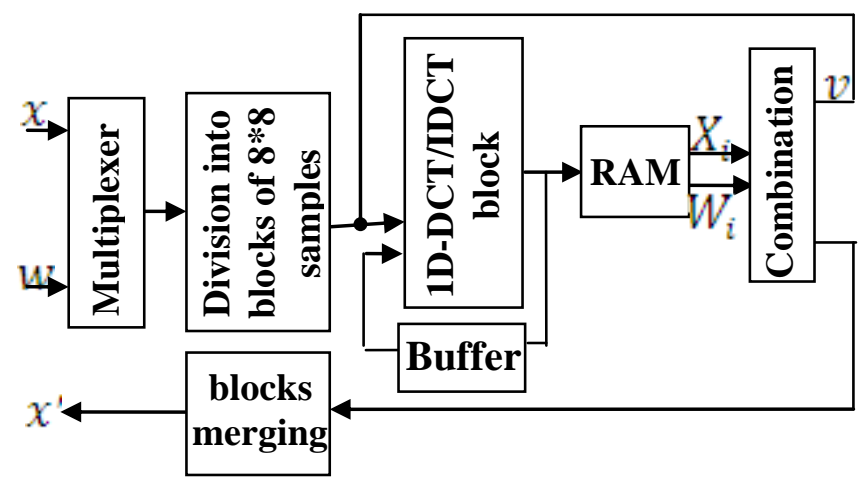

(b)The Proposed watermarking Scheme

Figure 2: The 2D-DCT Watermarking Scheme

The proposed architecture shown in figure 2.b has only one 1D-DCT/IDCT multiplierless block present in [12]. The 2-D DCT of the original image and that of the watermark performed one after another. As the amount of the computation of the latter is made much smaller than the former, the overall delay is not much larger than that of the two 2-D DCTs in parallel. The hardware consumption can thus, be reduced as Pie chart illustrated in Figure 3.a without sacrificing much the speed.

A look at RONI region in the original image a large number of adjacent pixels within the block has equal values. The 2D-DCT of these $(8 * 8)$ blocks is only DC element [13]. Using the DCT $(0,0)$ formula to calculate the 2D-DCT and leaving all other AC components. The omitted operations are more than $33.4 \%$ of total operation as verify in Figure 3.b.
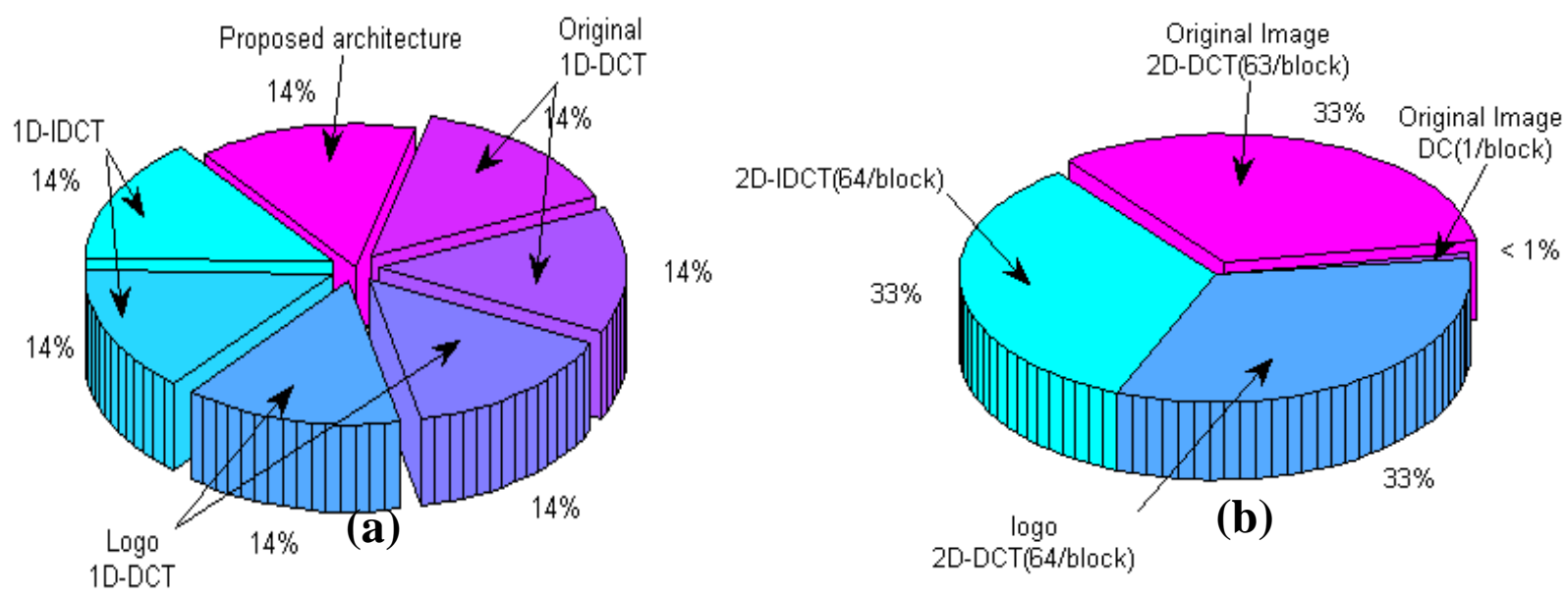

Figure 3: Pie charts (a)illustrates percents Hardware reduction (b)illustrates percents omitted operation in each $8 * 8 \mathrm{block}$

The proposed architecture work in three phases hav two steps, in the first step, calculate the 2D-DCT of Logo image, and in the second phase, only DC coefficient of original image blocks are calculated and stored in the RAM-memory. To apply equation (1) formula in combination stage, the Inverse Discrete Cosine Transform are done in last phase, all phases 
are done on the same unit. The LOGO embedding image are shown in Figure 4 for varying values of $\alpha$ and $\beta$.

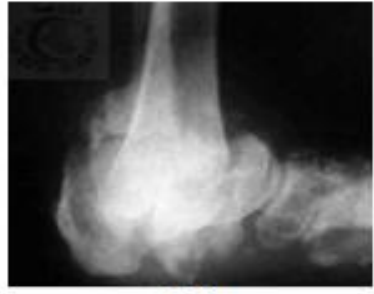

(a)

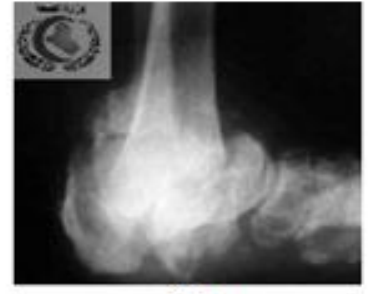

(b)

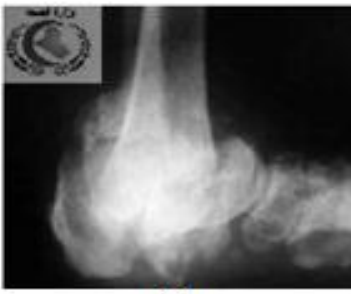

(c)

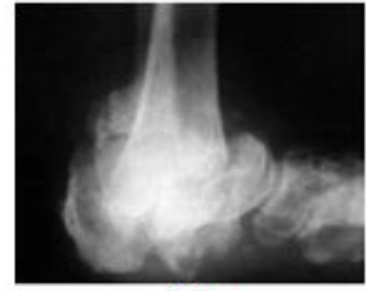

(d)

Figure 4. The images obtained after watermarking (a) for $\alpha=.97$ and $\beta=.07$ (b) for $\alpha=.97$ and $\beta=.27$ (c) for $\alpha=.97$ and $\beta=.47$ (d) invisible watermarking

\subsection{The Second Proposed Algorithm using LSB Replacement}

The second part of the proposed system is to embed the patient information inside X-ray host image, Our scheme is composed of two parts. The first one is to embed the patient's diagnosis in the medical image and the second is about how to extract it. This procedure must be carried out while guaranteeing the good quality of the image.

One of the most direct and simple technique is to embed the watermark code into the LSBs (Least Significant Bits) of the image. Since a change in LSB corresponds a change in 1 unit of image gray value, its modification is not perceivable by human eyes. The beauty of Xray images and all other medical images is that the LSBs for all pixels in the RONI are zeroes. The watermark is reversed by simply setting the LSBs of RONI back to zero.

The insertion method consists in carrying out a binary conversion then we divide the image into 8 plans of bits. Next, replace the last plans with the four message field after inverted binary bits .This function gives a message which we insert in LSB plan. Finally, we rebuild the 8 plans to lead to the watermarked image that is shown in figure 5 .

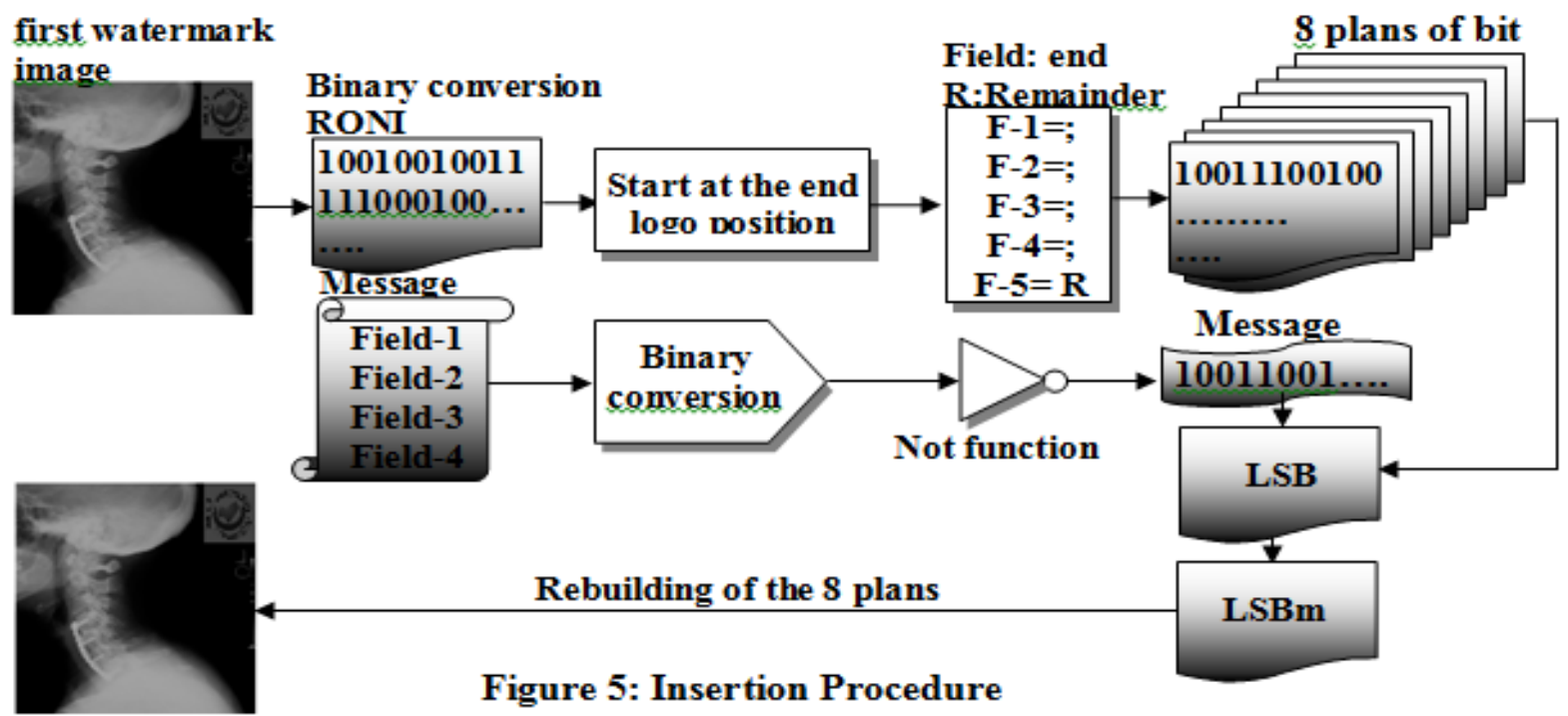

The following doctor embeds his diagnosis in the medical image received from the previous doctors by using the same procedure. The diagram insertion of the following doctors is like figure 6 which we replace the original image by the watermarked image. The patient 
information are extract by construct inversion LSBs, converted to ascii code and store as text file.

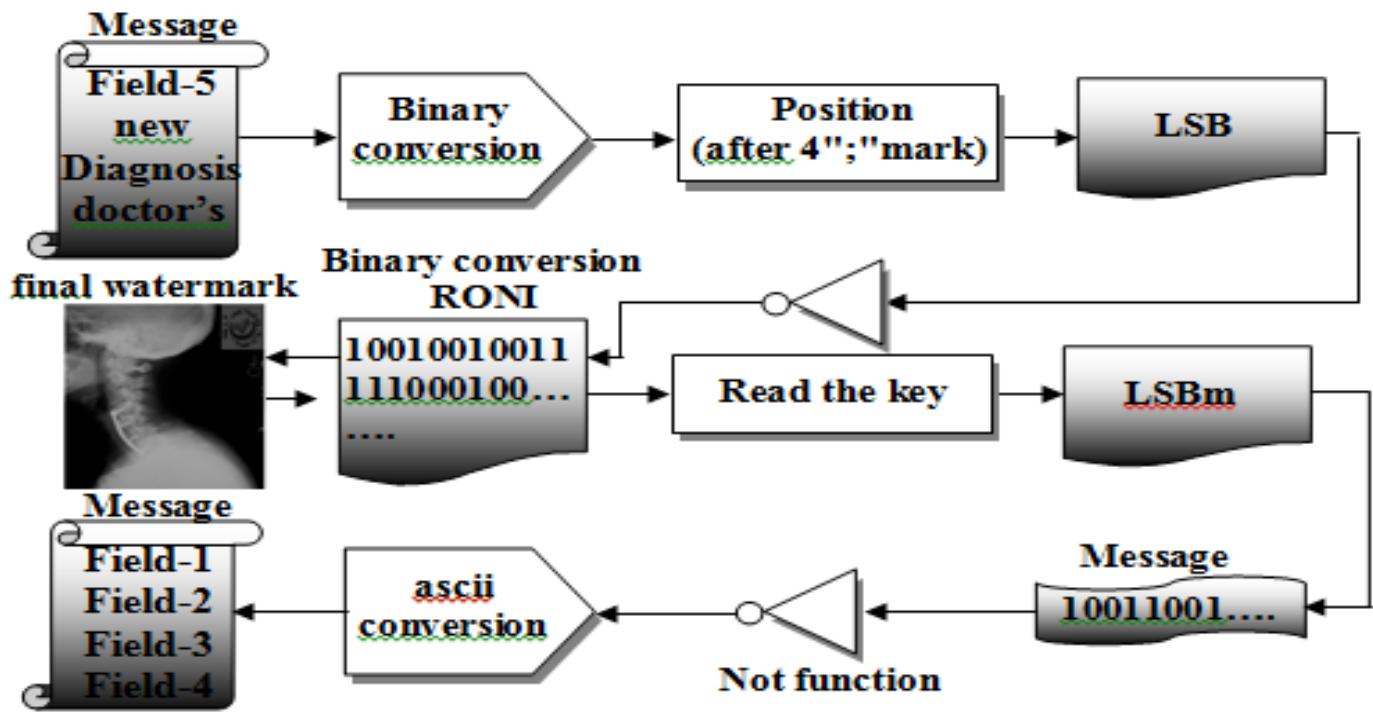

Figure 6: Patient data extraction and new diagnosis insertion

\subsection{Experimental Results:}

The proposed reversible watermarking scheme are implemented, and have successfully applied to many test images with different sizes and complexities as shown in Figure 7 . Equality between the original images and restored images has proved the reversibility of the proposed scheme in experiments. In our experiments, we take Iraqi health ministry LOGO and adopt peak signal-to-noise ratio (PSNR) value and byte number as measurements of image quality and embedding capacity, respectively as written in table 1.

Table 1: Comparison Results in terms of the Capacity (bits) and the PSNR value (dB) for different medical images.

\begin{tabular}{|c|c|c|c|c|c|c|}
\hline Image & $\begin{array}{c}\text { Image size } \\
\text { (Pixel) }\end{array}$ & threshold & $\begin{array}{c}\text { Capacity } \\
\text { (bit) }\end{array}$ & $\begin{array}{c}\text { PSNR } \\
\text { (dB) }\end{array}$ & $\begin{array}{c}\text { Embedded } \\
\text { (bit) }\end{array}$ & $\begin{array}{c}\text { Error } \\
\text { (embed - } \\
\text { extract)data }\end{array}$ \\
\hline Image_1 & $867 * 574$ & 0.4 & 310618 & 48.7 & 728 & 0 \\
\hline Image_2 & $425 * 400$ & 0.2745 & 100923 & 46.6 & 848 & 0 \\
\hline Image_3 & $604 * 516$ & 0.2909 & 184597 & 47.3 & 800 & 0 \\
\hline Image_4 & $800 * 420$ & 0.3843 & 62650 & 45.1 & 960 & 0 \\
\hline Image_5 & $738 * 600$ & 0.2627 & 200657 & 46.4 & 984 & 0 \\
\hline Image_6 & $740 * 539$ & 0.2745 & 218519 & 45.9 & 880 & 0 \\
\hline Image_7 & $867 * 574$ & 0.3176 & 1351307 & 47.7 & 896 & 0 \\
\hline Image_8 & $480 * 640$ & 0.3686 & 171825 & 46.6 & 736 & 0 \\
\hline Image_9 & $439 * 468$ & 0.3451 & 117075 & 44.9 & 832 & 0 \\
\hline
\end{tabular}

\section{The Proposed FPGA Architecture.}

The architecture of the encoder-watermarking chip is shown in Figure 8 consists of three distinct modules: LOGO DCT insertion module, Patient Information LSB module, and controller module. The structures of the extraction and insertion modules are very similar, 
due to uses of same components. The 1D-DCT multiplierless module present in [12], two multiplexers, one adder, two accumulators, three comparator and latches for pipeline implementation. The lower multiplexer (MUX) is used to choose between the watermarking strength factors, medical image and LOGO.

The top multiplexer helps in selecting an copy RONI or LOGO insertion process of watermarking insertion. The same block uses for 1D-DCT and 1D-IDCT, this architecture provides the capability to watermark a DCT block with minimum FPGA resources, latches are used for temporary buffering.

Buffer circuitry is used to assist in finding the transpose. It also serves as temporary storage for the first 1D DCT/IDCT coefficient, added control signal to select between DCT and IDCT operation. The DCT module does not have a separate controller; i. e. the main controller controls it. The controller is modeled as a synchronizer between modules. The key ( $\alpha$ and $\beta$ )are read from storage to the input register for their DCT coefficients to be calculated. The binary form of medical image is stored in memory after compare with appropriate threshold by comparator.

The structure of the decoder chip is shown in Figure 9 that have the same 1DDCT/IDCT modules, it starts operations when read the embed LOGO position key form the last 20 frame bits. After complete LOGO extraction, it compares with origin LOGO input and output one bit to check similarity. The LSB extraction uses LUT to convert the binary reconstructed bytes to ASCII code as well as insert the new diagnosis at the last field.

\section{Implementation Results}

The prototype is implemented in VHDL and synthesized using Xilinx Spartan-3E technology with an XC3S500-5fg320 target device. In order to provide a numeric example, we calculated the number of the calculation units, such as multipliers and adders, needed for mapping the proposed architecture with other references, the results are presented in Table 2. One can see that the proposed-DCT/IDCT-block structure needs only approximately $33.9 \%$ of the hardware of that of the one-DCT-blocks present in [8]. The increase of the time delay resulting from replacing the two-DCT-blocks by the one DCT structure is about twice, to dramatically parallel work as LSB procedure still busy.

Table 2: Comparison of Hardware Consumption and the time delay.

\begin{tabular}{|c|c|c|c|c|c|}
\hline & Single Ref[8] & One Ref[8] & Ref[7] & \multicolumn{2}{c|}{ Proposed Structure } \\
\hline & encoder & encoder & encoder & encoder & decoder \\
\hline function & LOGO only & LOGO only & LOGO only & L\&P & L\&P \\
\hline Multipliers & 42 & 22 & 16 & 0 & 0 \\
\hline Adders & 72 & 37 & 16 & 24 & 24 \\
\hline Latency & $64 \mathrm{~T}$ & $86 \mathrm{~T}$ & $163 \mathrm{~T}$ & $193 \mathrm{~T}$ & $193 \mathrm{~T}$ \\
\hline Clock(MHZ) & 1 & 1 & 1 & 50 & 50 \\
\hline Frequency & Not given & Not given & 83.996 & $79.789(\mathrm{MHZ})$ & $80.89(\mathrm{MHZ})$ \\
\hline Throughput & Sample/clock & Sample/clock & $\begin{array}{c}2 \\
\text { Sample/clock }\end{array}$ & Sample/clock & Sample/clock \\
\hline
\end{tabular}

$\mathrm{T}$ is the minimum interval between the two consecutive output samples depend on target

FPGA , L\&P=LOGO and Patient data. 


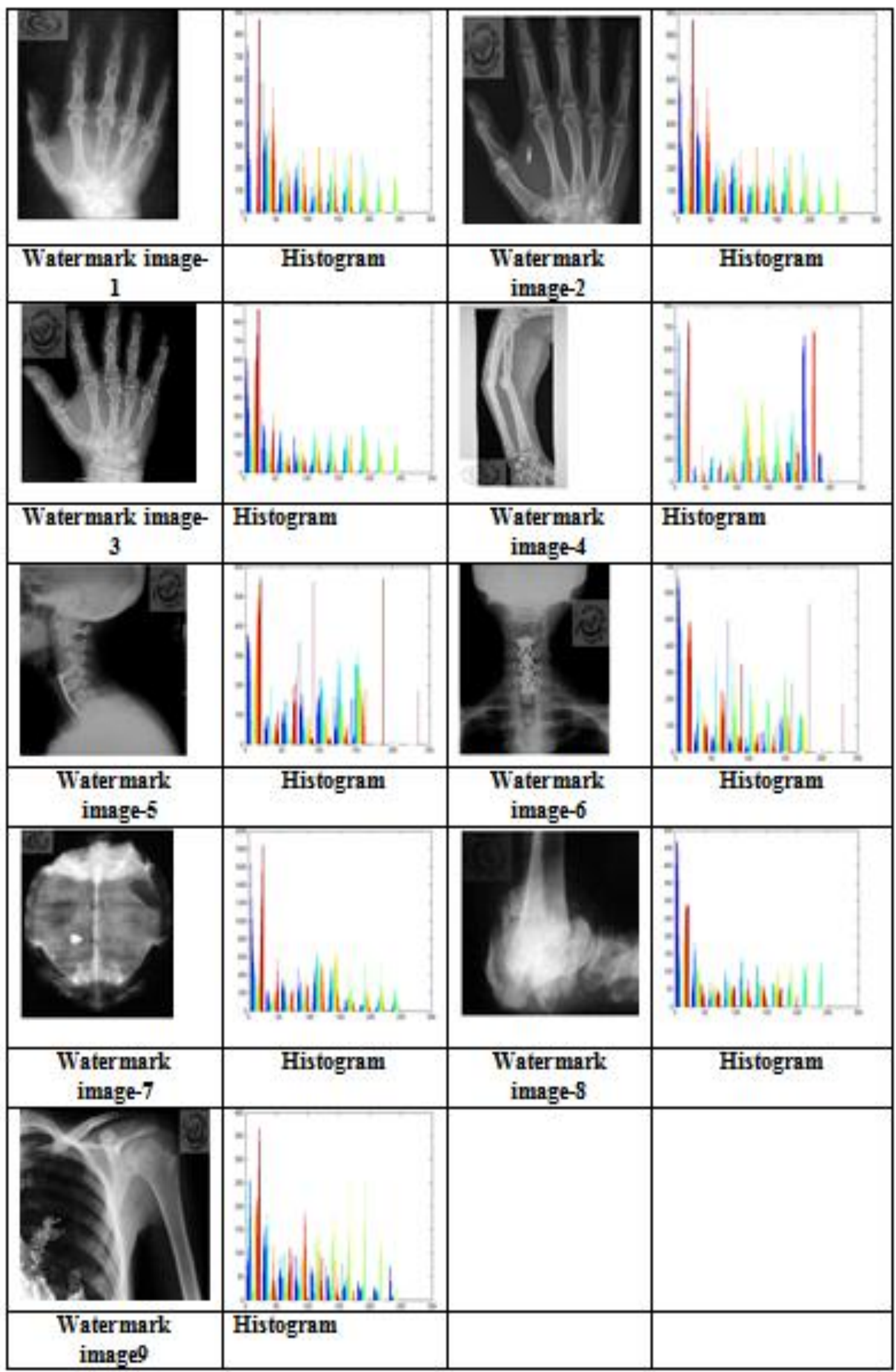

Figure 7: Watermarked versions of test images $(\alpha=.97$ and $\beta=.27)$ 


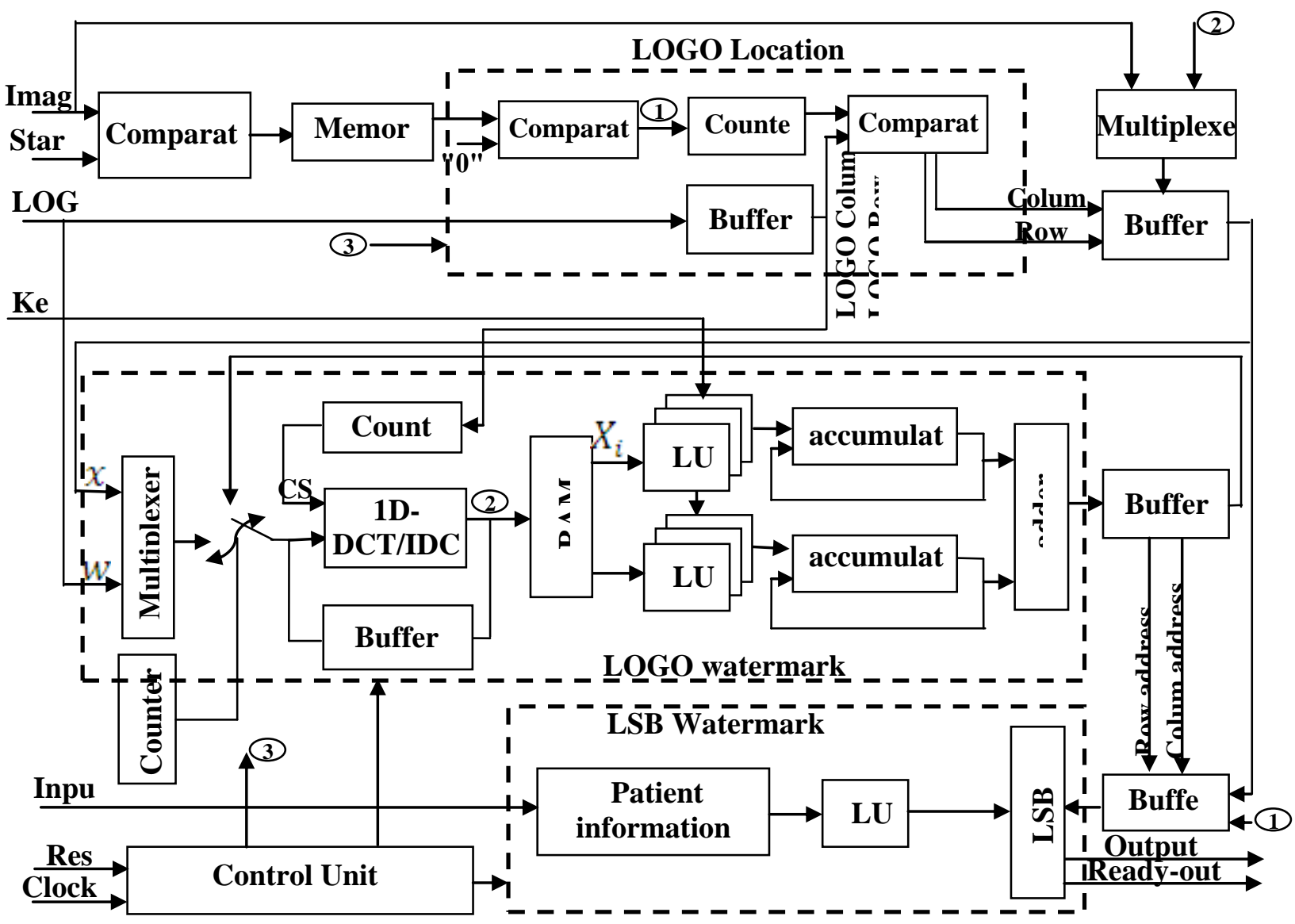

Figure 8: Architecture of the Encoder Chip

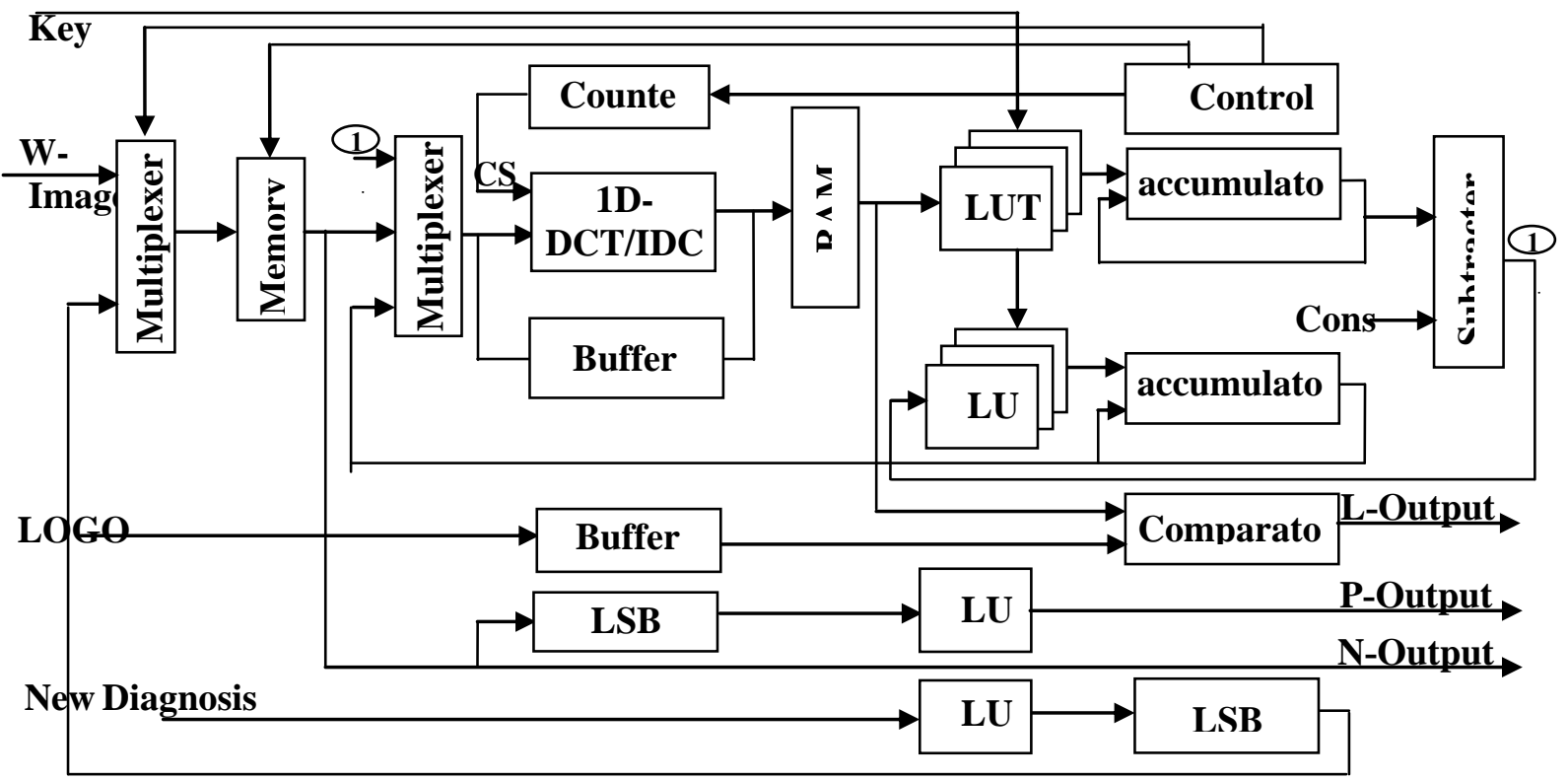

Figure 9: Architecture of the Decoder Chip 


\section{Conclusions}

This paper presented an FPGA Implementation of Electronic Patient Record system for multimedia communication between doctors and information technologies to protect the privacy of patients' records.

A multiplierless computation structure of image watermarking in DCT domain has been presented. It employs only a single 1D-DCT block to perform the 2-D DCT computation of the original image and that of the watermark in a serial manner to reduce the hardware consumption in circuit implementation.

According to the experimental results, the proposed reversible scheme provides a higher capacity and achieves better image quality for watermarked images. In addition, the power consumption is small and it operates in higher frequency greater than $100 \mathrm{MHZ}$ with one sample per clock throughput

\section{References:}

[1] S. Kaur, O. Farooq, R. Singhal and B. S. Ahuja," Digital watermarking of ECG data for secure wireless communication", International Conference on Recent Trends in Information, Telecommunication and Computing 12-13 March 2010, Kochi, Kerala, India , PP 140-144.

[2] M. Kallel, J. Lapayre and M. S. Bouhlel," A multiple Watermarking Scheme for Medical Image in the Spatial Domain", GVIP Journal, Volume 7, Issue 1, April, 2007, PP.37-42.

[3] L. Luo, Z. Chen, M. Chen, Xiao Zeng, and Z. Xiong, " Reversible Image Watermarking Using Interpolation Technique", IEEE Transactions on Information Forensics and Security, VOL. 5, NO. 1, March 2010, PP 187-193.

[4] S.-Chuin Liew and J. M. Zain," Reversible Medical Image Watermarking For Tamper Detection And Recovery", Computer Science and Information Technology (ICCSIT), 2010 3rd IEEE International Conference, Univ. Malaysia Pahang, 9-11 July 2010, PP 417-420.

[5] C. N. Gutierrez, G. Kakani, R. C.Verma and T. Wang," Digital Watermarking of Medical Images for Mobile Devices", IEEE International Conference on Sensor Networks, Ubiquitous, and Trustworthy Computing, 7-9 June 2010, Newport Beach, CA, USA, PP 421-425.

[6] C. Yao-yao,Z. Xin, X. Yong-liang, Y. Sheng and W. ling," An improved watermarking method based on double random phase encoding technique", Optics \& Laser Technology 42 (2010) , PP.617623. Journal home page: www.elsevier.com/locate/optlastec.

[7] R. K. Megalingam, V. Krishnan, V. Sarma, M. Mithun and R. Srikumar, "Hardware Implementation of Low Power, High Speed DCT/IDCT Based Digital Image Watermarking", International Conference on Computer Technology and Development, 13-15 Nov ,2009 , Kollam, India ,PP. 535-539.

[8] S. An and C. Wang," A Computation Structure for 2-D DCT Watermarking", This project has been supported by Natural Science and Engineering Research Council of Canada (NSERC),2009, PP.577-580.

[9] S. K. Bandyopadhyay and I. K. Maitra ," An Alternative Approach of Steganography using Reference Image", International Journal of Advancements in Technology http://ijict.org/ ISSN 09764860, Vol 1, No 1 (June 2010), PP.95-102.

[10] A. M. Zuhair and C. M. Yousuf, " FPGA Based Image Security and Authentication in Camera Using Invisible Watermarking Technique" , International Journal of Engineering Science and Technology Vol. 2(6), 2010, PP.1745-1751.

[11] Q. Hu, Z. Hou, and W. L. Nowinski, "Supervised Range-Constrained Thresholding", IEEE Transactions on Image Processing, VOL. 15, NO. 1, January 2006, PP. 228-240.

[12] A. F. mahmood and A. M. salah,"A Modified Color JPEG CODEC System Using inter pixel redundancy", accepted in Al-Rafidian Engineering journal.

[13] A. F. mahmood and A. M. salah ,"FPGA Implementation of Multiplierless DCT/IDCT Chip", accepted in Al-Rafidian Engineering journal.

The work was carried out at the college of Engineering. University of Mosul 